\title{
The Utility Premium of Friedman and Savage, Comparative Risk Aversion, and Comparative Prudence
}

May 18, 2015

\section{Introduction}

Recently, the literature has paid significant attention to the concept of utility premium. A risk's utility premium is defined as the loss in expected utility caused by the risk. This concept was introduced by Friedman and Savage (1948) more than sixty years ago; however, it has been largely ignored by the literature with the rare exception of Hanson and Menezes' study (1971), which promotes the concept in a discussion of the prudence notion.

Eeckhoudt and Schlesinger (2006) are among the first to rediscover the power of this concept and use it to show that the direction of preference for a particular class of lottery pairs is equivalent to signing a utility function's $n$th derivative. Eeckhoudt et al. (2007) use it to study the link between the signs of cross derivatives of a utility function and individual preference within a particular class of simple lotteries. Crainich and Eeckhoudt (2008) use it to establish a new measure for the intensity of downside risk aversion. 
Eeckhoudt and Schlesinger (2009) explain in detail the relevance of this concept for decision making under risk. Denuit and Eeckhoudt (2010a, b) use the concept to give a general foundation for higher order Arrow-Pratt risk aversion measures and develop some stronger measures of higher-order risk attitudes. More recently, Liu and Meyer (2013) use the ratio of utility premiums to examine the partial order of nth-degree Ross more risk averse while Crainich et al. (2013) use the concept of utility premium to characterize the preferences of agents who they call (mixed) risk lovers. ${ }^{1}$

In this short article we show that the utility premium normalized by the marginal utility is explained by the Arrow-Pratt risk aversion measure in exactly the same way as the risk premium of Pratt (1964), that is, the greater the risk aversion measure, the greater a risk's utility premium normalized by the marginal utility.

Moreover, we show that the concept of utility premium can also be used to explain comparative prudence. More precisely, we show that the greater the prudence measure, the greater the utility premium for disaggregating a certain loss of wealth and a zero-mean risk normalized by the utility function's second derivative.

The structure of the remaining paper is as follows. In Section 2, we introduce the concept of utility premium. In Section 3, we use utility premium to explain comparative risk aversion. In Section 4, we use utility premium to explain comparative prudence. Section 5 concludes the paper.

\footnotetext{
${ }^{1}$ Other related studies which use the concept of utility premium include Menegatti (2007) and Jindapon (2010).
} 


\section{The Utility Premium}

Throughout this paper, we assume that utility functions are strictly increasing, strictly concave, and thrice continuously differentiable. Given a von Neumann-Morgenstern utility function $u(x)$, we denote the Arrow-Pratt absolute risk aversion measure by $R(x)$, i.e., $R(x)=-u^{\prime \prime}(x) / u^{\prime}(x)$. Given a risk $\tilde{\epsilon}$, its utility premium is defined as $u(w)-E u(w+\tilde{\epsilon})$. As in Crainich and Eeckhoudt (2008), we normalize the utility premium by the marginal utility at the initial wealth: $\frac{u(w)-E u(w+\tilde{\epsilon})}{u^{\prime}(w)}$.

\section{Comparative Risk Aversion}

Let $u(w)$ and $v(w)$ be two utility functions with Arrow-Pratt absolute risk aversion measures $R_{u}(w)$ and $R_{v}(w)$ respectively. First consider a small zero-mean risk with variance $\sigma_{\epsilon}^{2}$. Applying a second order approximation, we obtain $\frac{u(w)-E u(w+\tilde{\epsilon})}{u^{\prime}(w)}=0.5 R(w) \sigma_{\epsilon}^{2}$. If, at a point $w, R_{u}(w)>R_{v}(w)$, for such a small risk, we have $\frac{u(w)-E u(w+\tilde{\epsilon})}{u^{\prime}(w)}>\frac{v(w)-E v(w+\tilde{\epsilon})}{v^{\prime}(w)}$. We show below that the global property also holds.

Proposition 1 For $\forall w, R_{u}(w) \geq\left[>_{a . e .}\right] R_{v}(w)$ if and only if $\forall w, \forall \tilde{\epsilon}$,

$$
\frac{u(w)-E u(w+\tilde{\epsilon})}{u^{\prime}(w)} \geq[>] \frac{v(w)-E v(w+\tilde{\epsilon})}{v^{\prime}(w)} .
$$

Proof: We first prove the "only if" part. We have

$$
\frac{v^{\prime}(w+x)}{v^{\prime}(w)}-\frac{u^{\prime}(w+x)}{u^{\prime}(w)}=\frac{u^{\prime}(w+x)}{v^{\prime}(w)}\left[\frac{v^{\prime}(w+x)}{u^{\prime}(w+x)}-\frac{v^{\prime}(w)}{u^{\prime}(w)}\right] .
$$

As $\left[\ln \frac{v^{\prime}(y)}{u^{\prime}(y)}\right]^{\prime}=R_{u}(y)-R_{v}(y) \geq\left[>_{a . e .}\right] 0$, from the above equation, $\frac{u^{\prime}(w+x)}{u^{\prime}(w)}-$ $\frac{v^{\prime}(w+x)}{v^{\prime}(w)}$ always has [strictly] the same sign as $x$. Therefore, since the direction 
of integration cancels out the sign, we have, for $\epsilon$,

$$
\int_{0}^{\epsilon}\left[\frac{v^{\prime}(w+x)}{v^{\prime}(w)}-\frac{u^{\prime}(w+x)}{u^{\prime}(w)}\right] d x \geq[>] 0
$$

which is equivalent to

$$
\frac{v(w+\epsilon)-v(w)}{v^{\prime}(w)}-\frac{u(w+\epsilon)-u(w)}{u^{\prime}(w)} \geq[>] 0
$$

Taking expectations with respect to $\epsilon$, we obtain the second inequality of the proposition.

To prove the "if" part, by contradiction, suppose this is not true. Given that $u(x)$ and $v(x)$ are twice continuously differentiable, there must exist an interval $[a, b]$ in which $R_{u}(x)<[\leq] R_{v}(x)$. By restricting the support of $\tilde{\epsilon}$ such that the support of $w+\tilde{\epsilon}$ is contained in $[a, b]$, we would obtain $\frac{u(w)-E u(w+\tilde{\epsilon})}{u^{\prime}(w)}<[\leq] \frac{v(w)-E v(w+\tilde{\epsilon})}{v^{\prime}(w)}$, contradicting the initial assumption that $\frac{u(w)-E u(w+\tilde{\epsilon})}{u^{\prime}(w)} \geq[>] \frac{v(w)-E v(w+\tilde{\epsilon})}{v^{\prime}(w)}$. Q.E.D.

We may relate this result to Pratt's (1964) result on risk premium and comparative risk aversion. Given a risk $\tilde{\epsilon}$, the risk premium $\pi(w, \tilde{\epsilon})$ at wealth $w$ is defined implicitly via $u(w+E \tilde{\epsilon}-\pi(w, \tilde{\epsilon}))=E u(w+\tilde{\epsilon})$. He shows that $\forall w, R_{u}(w) \geq\left[>_{a . e .}\right] R_{v}(w)$ if and only if $\forall w$ and $\forall \tilde{\epsilon}, \pi_{u}(w, \tilde{\epsilon}) \geq[>] \pi_{v}(w, \tilde{\epsilon})$. We have shown here that the utility premium of a risk normalized by the marginal utility is explained by the Arrow-Pratt risk aversion measure in exactly the same way as the risk premium.

\section{Comparative Prudence}

In this section we show that the concept of utility premium can also be used to explain comparative prudence. We first recall two lotteries defined by Eeckhoudt and Schlesinger (2006). Given $k>0$ and a zero-mean risk $\tilde{\epsilon}$, let 
$B_{3}=[-k ; \tilde{\epsilon}]$ and $A_{3}=[0 ; \tilde{\epsilon}-k]$ be two binary lotteries, the outcomes of which are assumed to have equal probability. ${ }^{2}$

As is explained by Crainich et al. (2013), for a risk-averter, both a certain loss in wealth $-k$ and a zero-mean risk $\tilde{\epsilon}$ are bad outcomes. So the lottery $A_{3}$ combines the two bad outcomes while the lottery $B_{3}$ disaggregates the two bad outcomes, and

$$
u(w-k)+E u(w+\tilde{\epsilon})-E u(w-k+\tilde{\epsilon})-u(w)
$$

is the utility premium for disaggregating the two bad outcomes.

Let $P_{u}(x)$ and $P_{v}(x)$ denote the absolute prudence of $u(x)$ and $v(x)$ respectively. We have the following result.

Proposition 2 Assume that $u(x)$ has positive third derivative. For $\forall w$, $P_{u}(w) \geq\left[>_{a . e .}\right] P_{v}(w)$ if and only if $\forall x, \forall k>0$, and $\forall$ zero-mean $\tilde{\epsilon}$,

$$
\begin{aligned}
& \frac{E u(x-k+\tilde{\epsilon})-u(x-k)-E u(x+\tilde{\epsilon})+u(x)}{u^{\prime \prime}(x)} \\
& \geq[>] \frac{E v(x-k+\tilde{\epsilon})-v(x-k)-E v(x+\tilde{\epsilon})+v(x)}{v^{\prime \prime}(x)} .
\end{aligned}
$$

Proof: We only prove the non-strict version of the result. We first prove the "only if" part. From Proposition $1, P_{u}(x) \geq P_{v}(x)$ implies for all $\tilde{\epsilon}{ }^{3}$

$$
\frac{v^{\prime \prime}(x)}{u^{\prime \prime}(x)}\left[E u^{\prime}(x+\tilde{\epsilon})-u^{\prime}(x)\right] \geq\left[E v^{\prime}(x+\tilde{\epsilon})-v^{\prime}(x)\right]
$$

It follows that for all $k>0$,

$$
\int_{y-k}^{y} \frac{v^{\prime \prime}(x)}{u^{\prime \prime}(x)}\left[E u^{\prime}(x+\tilde{\epsilon})-u^{\prime}(x)\right] d x \geq \int_{y-k}^{y}\left[E v^{\prime}(x+\tilde{\epsilon})-v^{\prime}(x)\right] d x .
$$

\footnotetext{
${ }^{2}$ Eeckhoudt and Schlesinger (2006) use the two lotteries to characterize prudence.

${ }^{3}$ This is obtained by replacing $u(x)$ and $v(x)$ by $-u^{\prime}(x)$ and $-v^{\prime}(x)$ respectively in the proposition.
} 
In the meantime, as $P_{u}(x) \geq P_{v}(x), \frac{v^{\prime \prime}(x)}{u^{\prime \prime}(x)}$ is increasing. This implies for $x \in[y-k, y], \frac{v^{\prime \prime}(x)}{u^{\prime \prime}(x)} \leq \frac{v^{\prime \prime}(y)}{u^{\prime \prime}(y)}$. Moreover, since zero-mean $\tilde{\epsilon}$ and convex $u^{\prime}(x)$ imply $E u^{\prime}(x+\tilde{\epsilon})-u^{\prime}(x) \geq 0, \int_{y-k}^{y}\left[E u^{\prime}(x+\tilde{\epsilon})-u^{\prime}(x)\right] d x \geq 0$, and it follows that

$$
\frac{v^{\prime \prime}(y)}{u^{\prime \prime}(y)} \int_{y-k}^{y}\left[E u^{\prime}(x+\tilde{\epsilon})-u^{\prime}(x)\right] d x \geq \int_{y-k}^{y}\left[E v^{\prime}(x+\tilde{\epsilon})-v^{\prime}(x)\right] d x,
$$

which is equivalent to Inequality (1). This proves the "only if" part.

The converse statement can be proved using a standard type of argument by contradiction similar to that for Proposition 1. Q.E.D.

We give three remarks. (i) As was pointed out in the proof, since $u(x)$ has positive third derivative, $E u(x-k+\tilde{\epsilon})-u(x-k)-E u(x+\tilde{\epsilon})+u(x)=$ $-\int_{y-k}^{y}\left[E u^{\prime}(x+\tilde{\epsilon})-u^{\prime}(x)\right] d x \leq 0$. As agents are risk averse, this implies that the left-hand side of Inequality (1) is always positive.

(ii) As was explained earlier, the numerator of the left [right] hand side of Inequality (1) is the negative utility premium for disaggregating the two bad outcomes $\tilde{\epsilon}$ and $-k$ by $u(x)[v(x)]$. Thus the proposition tells us that the greater the prudence measure the greater the utility premium for disaggregating the two bad outcomes normalized by the negative second derivative of the utility function.

(iii) The above result gives a different characterization of the prudence measure from the one given by Chiu (2005). Chiu shows that an agent has greater prudence than a reference agent if and only if his expected utility is increased by a class of compensated downside risk increases which preserve the expected utility of the reference agent.

It is interesting to relate this proposition to a result given by Crainich and Eeckhoudt (2008) who use the same utility premium to explain the downside risk aversion measure $u^{\prime \prime \prime} / u^{\prime}$. In their result they normalize the 
utility premium by the marginal utility, and show that when the risk is small, the greater the ratio $u^{\prime \prime \prime} / u^{\prime}$, the greater the utility premium normalized by the marginal utility. From the proposition we can obtain a sufficient condition for an agent to have greater utility premium for disaggregating the two bad outcomes normalized by the marginal utility than another agent in the general case where the risk may not be small.

Corollary 1 Assume that $u(x)$ has positive third derivative. If for $\forall w$, $P_{u}(w) \geq P_{v}(w)$ and $R_{u}(w) \geq R_{v}(w)$, then for $\forall w, \forall k>0$, and $\forall$ zero-mean $\tilde{\epsilon}$

$$
\begin{aligned}
& -\frac{E u(x-k+\tilde{\epsilon})-u(x-k)-E u(x+\tilde{\epsilon})+u(x)}{u^{\prime}(x)} \\
& \geq-\frac{E v(x-k+\tilde{\epsilon})-v(x-k)-E v(x+\tilde{\epsilon})+v(x)}{v^{\prime}(x)} .
\end{aligned}
$$

Proof: As earlier, we use $S_{u}$ and $S_{v}$ to denote the left and right hand sides of the Inequality (1) respectively. Then the left and right hand sides of the Inequality (2) are equal to $S_{u} R_{u}(w)$ and $S_{v} R_{v}(w)$ respectively. Since for $\forall w$, $P_{u}(w) \geq P_{v}(w)$, from Proposition 2 , we have Inequality (1), i.e., $S_{u} \geq S_{v}$. In the meantime we have, for $\forall w, R_{u}(w) \geq R_{v}(w)$. Noting that from Remark (i) on Proposition $2, S_{u} \geq 0$, we immediately obtain Inequality (2). Q.E.D.

\section{Conclusion}

In this paper, we have shown that the utility premium of a risk normalized by the marginal utility is explained by the Arrow-Pratt risk aversion measure in exactly the same way as the risk premium of Pratt. This bridges the gap between the utility premium and the risk premium and gives an alternative characterization of Arrow-Pratt risk aversion. We have also shown 
that the greater the prudence measure, the greater the utility premium for disaggregating a certain loss of wealth and a zero-mean risk normalized by the utility function's second derivative.

These results not only improve the understanding of comparative risk aversion and comparative prudence, but also improve the understanding of the concept of the utility premium, which has shown to be a useful tool for research in decision making under risk. 


\section{REFERENCES}

1. Crainich, D., and Eeckhoudt, L., "On the Intensity of Downside Risk Aversion," Journal of Risk and Uncertainty 36, 267-276, 2008.

2. Crainich, D., Eeckhoudt, L., and Trannoy A. "Even (Mixed) Risk Lovers Are Prudent," American Economic Review 103, 1529-1535, 2013.

3. Chiu, W. H. "Skewness Preference, Risk Aversion, and the Precedence Relations on Stochastic Changes," Management Science 12, 1816-1828, 2005.

4. Denuit, M., and Eeckhoudt, L., "A General Index of Absolute Risk Attitude," Management Science 56, 712-715, 2010a.

5. Denuit, M., and Eeckhoudt, L., "Stronger Measures of Higher-order Risk Attitudes," Journal of Economic Theory 145 2027-2036, 2010b.

6. Eeckhoudt, L., Rey, B., and Schlesinger, H., "A Good Sign for Multivariate Risk Taking," Management Science 53, 117-124, 2007.

7. Eeckhoudt L., and Schlesinger H., "Putting Risk in Its Proper Place," American Economic Review 96, 280-289, 2006.

8. Eeckhoudt, L., and Schlesinger, H., "On the Utility Premium of Friedman and Savage," Economics Letters 105, 46-48, 2009.

9. Friedman, M., and Savage, L., "The Utility Analysis of Choices Involving Risk," Journal of Political Economy 56, 279-304, 1948.

10. Hanson, D. L., and Menezes, C. F., "On a Neglected Aspect of the Theory of Risk Aversion,” Western Economic Journal 9, 211-217, 1971. 
11. Jindapon, P. "Prudence Probability Premium," Economic Letters 109, 34-37, 2010.

12. Liu, L., and Meyer, J., "Substituting One Risk Increase for Another: A Method for Measuring Risk Aversion," Journal of Economic Theory 148, 2706-2718, 2013.

13. Menegatti, M. "A New Interpretation for the Precautionary Saving Motive: A Note," Journal of Economics 92, 275-280, 2007.

14. Pratt, J. "Risk Aversion in the Small and in the Large," Econometrica $32122-136,1964$. 